\title{
Ni esa pretensión ni esa modestia (una revista de teoría y sus fantasías de intervención)
}

\author{
- Analía Gerbaudo / Universidad Nacional del Litoral - CONICET \\ analia.gerbaudo@conicet.gov.ar
}

L'institution instituée fait oublier qu'elle est issue d'une longue série d'actes d'institution et se présente avec toutes les apparences du naturel. Pierre Bourdieu, Raisons pratiques. Sur la théorie de l'action

En 1993, durante un Simposio sobre «Desconstrucción y pragmatismo» realizado en el Collège International de Philosophie de París del que participaron Ernesto Laclau, Chantal Mouffe, Richard Rorty y Simon Chritchley, Jacques Derrida inicia su conferencia con una confesión, en principio, desconcertante: «soy muy sentimental y creo en la felicidad, y pienso que eso tiene un papel determinante en mi obra» (1996: 15I). ¿Por qué elegir este comienzo autobiográfico para encarar una respuesta a un debate sobre el lazo entre desconstrucción, fronteras entre lo privado y lo público, literatura y democracia? ¿Cómo leer, además, ésta y cualquier otra afirmación autobiográfica, después de todos los pliegues (muy a su pesar: endeudados con el psicoanálisis), entre los que Derrida ha sabido resguardar afirmaciones de este tenor, con independencia de quién las profiera?: «nadie sabrá jamás a partir de qué secreto escribo y que yo lo diga no cambia nada» (Derrida I99Ia:218), prevenía en "Circonfesión». Alerta que con cautela, Benoît Peeters coloca como epígrafe a su descomunal biografía en un gesto que puede equipararse al «lo sé, lo sé, pero igual lo hago».

Desde obstinaciones parecidas a la de Peeters, se pone a circular este segundo número de El taco en la brea, empeńado en interrogar (o en no olvidar) qué le pone sentido a nuestros andares cotidianos. Entre ellos, escribir un artículo en español para una revista centrada en problemas de teoría literaria.

En el editorial para el último número de Punto de vista, Beatriz Sarlo afirma que el sentido de esos treinta ańos de trabajo que, entre otras cosas, habían dado como resultado noventa números, no había estado puesto en la «actividad conservadora de recopilar buenos artículos» sino más bien en hacer «vir[ar] los ejes del debate» (2). No tenemos una pretensión tan alta (no podemos tenerla: hemos 
leído a Pierre Bourdieu — I987, I997- y algo sobre «agentes» y poder simbólico hemos aprendido en todos estos años). Tampoco la modestia de circunscribirnos a recopilar buenos artículos: esa es la exigencia mínima de arranque. En verdad a lo que se aspira es a contribuir en las discusiones del campo: el control de los artículos por el comité científico, el cuidado en la decisión de los temas alrededor de los que gira cada dossier, la elección de secciones que permitan relevar diferentes producciones (traducciones, reseńas, entrevistas, materiales de archivo, etc.) se conjugan para intentar alcanzar ese resultado.

En este sentido cabe destacar, en primer lugar, la continuidad entre el dossier preparado por Germán Prósperi y varias de las encendidas discusiones sostenidas en diferentes encuentros realizados en la Universidad Nacional del Litoral durante 20I4. Para empezar, el $x$ Congreso de Hispanistas: la historia de la institucionalización de nuestras disciplinas y de su enseñanza en diferentes niveles de nuestro sistema educativo, los nexos entre las literaturas argentina y española, las hibridaciones que impiden rápidas demarcaciones tanto entre géneros como entre territorios, los trabajos de la memoria (en este caso, inspirados en y a partir del teatro) se retoman en este Dossier donde resuenan, por otro lado, ecos de otras conversaciones. Entre ellas, las sostenidas con Marcelo Topuzian sobre teoría literaria, y con Laura Scarano sobre teoría literaria y poesía contemporánea durante sus respectivos Seminarios de posgrado; por otro lado, las sostenidas con Gloria Chicote durante sus asesorías técnicas a propósito de las también encendidas discusiones respecto de la posibilidad de crear en nuestra universidad una unidad de doble dependencia CONICET-UNL que articule nuestra producción en ciencias sociales y humanas. Finalmente, los diálogos con Marcela Arpes y Silvio Mattoni, sobre metodologías de la investigación "disciplinaria» y bordes «post» (posdisciplina y desconstrucciones del género fueron temas discutidos durante el II Coloquio de avances de investigaciones del CEDINTEL y también a través de un persistente intercambio de mails a propósito de una postulación científica).

Imposible no atender, en ese sentido, el deleuziano elogio de «lo menor» con que Scarano abre su trabajo para este número de la revista en cruce con la minuciosa historia institucional que reconstruye Chicote: todas las visitas, todas las presentaciones del 2014 estuvieron cruzadas por una discusión que tangencialmente, se expresa también en estos artículos. ¿A qué atender en ciencias humanas? ¿Cómo escribirlas? ¿En qué lengua? ¿Dónde publicar los resultados de nuestras investigaciones? ¿Para qué publicarlos? ¿Para qué lectores? ¿En qué soporte? Y enredando las preguntas anteriores en un «bucle extraño» (Hofstadter): ¿Podemos dar una respuesta unívoca, para todas sus líneas, aquí y ahora, al modo de un patrón?

No quiero desaprovechar la oportunidad de la presentación de este segundo número de nuestra revista que tiene como primeros lectores, a nuestro Comité Científico, es decir, a agentes que gestionan la producción en ciencias humanas y sociales en diversas instituciones de Argentina y del extranjero, sin dejar planteada una inquietud y una posición sobre este nudo de preguntas. 
Durante 2014 la autogestionada Comisión Interinstitucional de Elaboración de Criterios de evaluación para las Humanidades y Ciencias Sociales (CIECEHCS), pone a circular en formato digital un documento que resume criterios de evaluación de la producción científica en estos campos. Destaco del documento, por un lado, la atención a un conjunto de elementos diferenciales de las humanas y las sociales que valdrá la pena considerar si efectivamente luego pueden aplicarse al momento de realizar las ponderaciones; por otro lado, la importancia de la precisión de cuestiones de gestión nodales si se pretende un procedimiento democrático y democratizador de evaluación que promueva una ciencia creativa y no sujeta a pasillismos sobre las estrategias para obtener un subsidio (tema que también rondó nuestras charlas del año pasado a propósito de dos libros, uno de Laura Scarano y Aymará de Llano y otro de Miguel Dalmaroni que, en diferentes momentos, han contribuido a transparentar lógicas y prácticas sólo conocidas, en el tiempo de su publicación, por las universidades con tradición en el campo).

Sobre este último punto destaco que la comisión observa la necesidad de que los criterios y escalas de puntajes públicos estén accesibles tanto para evaluados como para evaluadores «antes del proceso de presentación de solicitudes y/o de evaluación» (Castorina y otros:3). Sobre la primera cuestión, el documento atiende no sólo a la cuantificación sino a la calidad de lo producido enfatizando, entre otros, en la «originalidad» de los resultados y en la consideración de si la propuesta enriquece las «metodologías de la investigación» (7): aspectos que refuerzan el estímulo a la imaginación y al riesgo en el ejercicio de la práctica. En relación con esta apuesta, la comisión se involucra con un aspecto controversial cuando afirma "que el factor de impacto de una revista no puede considerarse representativo de la calidad de un artículo» (7). Y agrega en nota al pie, retomando resultados de otros debates: «el contenido no puede confundirse con el envoltorio» (7). También sobre este punto profiere una afirmación polémica, en defensa, creo entrever, de la divulgación, la extensión y la enseñanza, cuando apela a no «priorizar sólo el rubro publicaciones» (7) pero además, cuando dentro de estas, recomienda enfáticamente «valorizar sus diferentes formatos como, por ejemplo, los audiovisuales que amplían las posibilidades de difundir la producción científica» (7).

Siguiendo con este punto, el rescate del libro es otro punto nodal, en especial para quienes trabajamos desde investigaciones cualitativas: «a diferencia del artículo, la mayor extensión del libro hace posible el desarrollo minucioso de un argumento así como la exposición y fundamentación de la evidencia empírica cualitativa» (8). Esto evita, como nos sucede a quienes estamos en la etapa final de un tramo de investigación largo, la autocita y el envío a artículos propios en función de evitar banalizar el contenido dado el espacio acotado de cualquier artículo y, por otro lado, permite apreciar «un esfuerzo de entre 5 y io años de trabajo" que "debe ser considerado en su valor cualitativo y no como una publicación de género menor con respecto al artículo» (8).

Entre otro núcleo de este punto está el idioma en el que se enuncia el resultado: junto a la ponderación de publicaciones en la lingua franca por los argu- 
mentos por todos conocidos, la comisión resalta que nuestras disciplinas suelen tener como destinatarios de sus resultados «a quienes son parte de problemáticas regionales y/o culturales» (Io) poniendo en duda la atención preferencial a lo declinado en inglés.

Sumando más ítems al debate, propone «valorar las reseñas críticas que tienen la dimensión y complejidad de un artículo (distinguiéndolas de los simples comentarios in extenso de las bondades de un libro)» (I2) dado que éstas "permiten el conocimiento de los trabajos de investigación a un público más amplio» y abren «el debate al interior de un área temática específica» contribuyendo a «la consolidación de un campo» y de las ciencias sociales y humanas en su conjunto (I2). Una valiosa contribución, además, sobre qué se espera de una reseña en el campo.

También pondera la participación en reuniones científicas «en tanto y en cuanto permita visualizar el proceso por el cual los investigadores someten al debate su producción en distintos momentos de su elaboración y ante audiencias diversas, tanto nacionales como internacionales» (I4): sobre este punto, otro gesto valiente consiste en admitir que «si bien algunas reuniones parecen más relevantes debido a su reconocimiento mundial, (...) lo cierto es que su actual masividad y sus tiempos extremadamente acotados de exposición, no permiten el menor intercambio entre investigador y audiencia»(I4). Otro aporte crucial ligado al cambio necesario de formatos que exigen las nuevas formas de presentación de los resultados, luego de la irrupción de Internet y de su alteración radical respecto de la circulación de la información.

Otro aspecto considerado es la importancia a la «sustentabilidad en el tiempo» de las prácticas de transferencia (I8). Un punto medular asociado a las resistencias y a la claridad respecto de las metas a alcanzar mediante la gestión institucional de todas y cada una de las prácticas a las que se apuesta.

Se me disculpará si la síntesis resulta abrumadora. Repongo estos puntos de vista porque abrigo la fantasía de que redifundirlos contribuirá a discutirlos y, por lo tanto, a actuar activamente en el delineado de la agenda científica por-venir. Muchos investigadores del CONICET nos asombramos ante algunas leyendas puestas en la evaluación de nuestros informes bianuales: ¿qué supone, no sólo acatar mansamente la recomendación de publicar en «revistas indexadas y de circulación internacional», sino focalizar especialmente allí nuestra producción? ¿Es esa «recomendación» excluyente, dicotómica, o resistirá la muy desconstruccionista lógica del «y .... ...»? ¿Sólo la pertenencia de una revista al «grupo I» o al «grupo 2» asegura que es el mejor lugar para difundir un resultado, todo resultado, cualquier resultado de cualquier rama de las ciencias humanas y en toda situación? ¿Es un valor, digamos, universal, general, abarcativo, omnicomprensivo? Y llevando un poco más allá la cuestión: ¿de qué hablamos cuando hablamos de «internacionalización» (Sapiro y otros, Heilbron y otros)? ¿Incluimos en el patrón la circulación Sur-Sur o sólo Sur-Norte? ¿O Norte-Sur? Y otra vez, volviendo al inicio: ¿cuál es el sentido de nuestras prácticas? ¿Para quién escribimos? ¿Para qué sostener una revista en teoría literaria declinada en español? ¿Qué credo nos 
impulsa? ¿Qué fantasías? ¿Y a quienes publican en ella? ¿Y a quienes nos apoyan desde el Comité Científico donándonos ese bien escaso, el tiempo, para evaluar nuestros envíos? Pregunto, como diría Horacio González, para saber «qué son esos nombres que pronunciamos» (IO9): «internacionalización», ¿libro versus revistas?, ¿inglés versus cualquier lengua?, ¿Sur versus Norte?, ¿América Latina versus Europa y EE. UU?, ¿papel versus digital? Si apunto estos binomios, me apuro en aclararlo, no es por capricho, sino porque han atravesado importantes debates sostenidos en foros y coloquios, también durante 20I4. Sospecho que hay menos acuerdo que el aparente sobre el tema y me preocupa la repercusión que nuestras posiciones tendrán en el futuro mediato, no sólo en nuestro trabajo individual sino muy especialmente en el de los jóvenes bajo nuestro cuidado (los «recursos humanos», digamos).

A propósito de ello, estamos discutiendo la posibilidad, en principio con algunos integrantes del Comité Científico y también con otros investigadores que trabajan en filosofía desconstruccionista, en destinar el próximo dossier de nuestra revista a tratar este problema, desplazando el que pensábamos dedicar a las «herencias derrideanas» (una deriva de las conversaciones mantenidas en el Coloquio Homenaje a Jacques Derrida "La soberania en cuestión» organizado, también en 20I4, en el Museo del libro y de la lengua por Ana Paula Penchaszadeh y Emmanuel Biset). Se verá. No sería desatinado imaginar, por ejemplo, un dossier en el que el Comité Científico de esta revista y otros investigadores interesados en dar su punto de vista, expusieran su posición: esos agentes del campo integran centros de investigación de las más variadas procedencias. No es desatinado aventurar que el cruce de miradas enriquecería...

Finalmente, y no sin relación con lo anterior, un aviso y un agradecimiento.

El aviso compromete el anuncio de una nueva sección de aparición «eventual» (como les llamamos en el primer número de El taco en la brea a las que aparecerán según la necesidad de comunicar algo). «Envíos» es el nombre elegido para la sección destinada a publicar «noticias» de traducciones en curso que, por negociación de derechos editoriales (cuya relación con los derechos de autor, como nos ha enseñado Anna Gargatagli, plantea más de un debate, dada su no necesaria homología), no se pueden poner a circular. Consignamos entonces un pasaje, no superior al límite permitido por la legislación argentina para una cita bibliográfica a los efectos de, por un lado, evitar problemas jurídicos y, por el otro, impulsar a leer el texto trayendo algo de su tono, de su respiración, de su fraseo, de sus ideas, sin la mediación de nuestras glosas (como sucedería en una reseña) aunque sí, con la de nuestra escritura. No conozco mayor gesto de amor a un libro que traducirlo, más cuando se es un traductor amateur, es decir, cuando lo que motiva la práctica es, centralmente, el puro deseo de un aporte que se considera crucial para el campo, se conozca.

El agradecimiento es, en primer lugar, para los colegas que a partir de este número se han sumado generosamente a cooperar como Comité Científico de $E l$ taco en la brea: Edda Hurtado, Delmiro Rocha, Raúl Rodríguez Freire, Andrea 
Pagni y Victoria Torres. También es para todos los que creen en nuestro trabajo al confiarnos el suyo: cuidar los criterios y los acuerdos pautados para la evaluación de cada artículo, para el control de cada reseńa, para comunicar la aceptación o el rechazo fundamentado de una propuesta es otro de nuestros modos de contribuir a una institucionalización (Sapiro, Schögler) «responsable» (Derrida I99ıb) de las ciencias humanas y, más concretamente, de la investigación literaria.

\section{Bibliografía}

Bourdieu, Pierre (1987). Choses dites. París: De Minuit. (1997). Méditations pascaliennes. París: Du Seuil.

Castorina, José Antonio y otros (2014). Criterios de evaluación de la producción cientifica de las Humanidades y Ciencias Sociales. Mimeo.

Chicote, Gloria (2015). «El hispanomedievalismo argentino». El taco en la brea 2.

Dalmaroni, Miguel (Dir.) (2009). La investigación literaria. Problemas iniciales de una práctica. Santa Fe: Universidad Nacional del Litoral.

Derrida, Jacques (I991a). «Circonfesión», en Jacques Derrida y Geoffrey Bennington. Jacques Derrida. Madrid: Cátedra, 1994, 25-318. Traducción de María Luisa Rodríguez Tapia. (199ib). L'autre cap. La démocratie ajournée. París: Minuit.

(1996). "Notas sobre desconstrucción y pragmatismo», en Chantal Mouffe, compiladora. Desconstrucción y pragmatismo. Buenos Aires: Paidós, I5I-I70. Traducción de Marcos Mayer.

González, Horacio (2008). El peronismo fuera de las fuentes. Buenos Aires: Universidad Nacional de General Sarmiento/Biblioteca Nacional.

Heilbron, Johan y otros (20I4). Handbook of Indicators of the Internationalization of the Social and Human Sciences. InTERCO SSH-EHess. Mimeo.

Hofstadter, Douglas (1979). Gödel, Escher, Bach. Un Eterno y Grácil Bucle. Barcelona: Tusquets, 1998. Traducción de Mario Usabiaga y Alejandro López Rousseau.

Peeters, Benoît (20Io). Derrida. París: Flammarion.

Sapiro, Gisèle (2012). International Cooperation in the Social Sciences and Humanities: Comparative Socio-Historical Perspectives and Future Possibilities. Proyecto. Consultado el $7 \mathrm{de}$ febrero de 2015 en http://www.interco-ssh.eu

SAPIRO, GISÈLE y otros (20I3). Indicators of the Internationalization of an academic discipline in Social Sciences and Humanities. InTERCo sSH-EHEss. Mimeo.

Sarlo, Beatriz (2008). «Final». Punto de vista 90, I-2.

Scarano, Laura (2015). "Poéticas de lo menor en el hispanismo transatlántico». El taco en la brea 2.

Scarano, Laura y Aymará de Llano (200I). Saberes de la escritura. Géneros y convenciones del discurso académico. Mar del Plata: Martín.

Schögler, Rafael (20I4). Handbook of Indicators of Institutionalization of Academic Disciplines in Social and Human Sciences. INTERCO sSH-EHESS. Mimeo. 